\title{
Verification and Semantic Parallelization of Goal-driven Autonomous Software
}

\author{
Damian Dechev $^{1}$, Nicolas Rouquette ${ }^{2}$, Peter Pirkelbauer ${ }^{1}$, and Bjarne Stroustrup ${ }^{1}$ \\ dechev@tamu.edu, nicolas.rouquette@jpl.nasa.gov, peter.pirkelbauer@tamu.edu, bs@cs.tamu.edu \\ Texas A\&M University ${ }^{1}$ \\ College Station, TX 77843-3112 \\ Jet Propulsion Laboratory, California Institute of Technology ${ }^{2}$ \\ 4800 Oak Grove Drive, M/S 301-270, Pasadena, CA
}

\begin{abstract}
Future space missions such as the Mars Science Laboratory demand the engineering of some of the most complex manrated autonomous software systems. According to some recent estimates, the certification cost for mission-critical software exceeds its development cost. The current processoriented methodologies do not reach the level of detail of providing guidelines for the development and validation of concurrent software. Time and concurrency are the most critical notions in an autonomous space system. In this work we present the design and implementation of a first concurrency and time centered framework for verification and semantic parallelization of real-time $\mathrm{C}++$ within the JPL Mission Data System Framework (MDS). The end goal of the industrial project that motivated our work is to provide certification artifacts and accelerated testing of the complex software interactions in autonomous flight systems. As a case study we demonstrate the verification and semantic parallelization of the MDS Goal Networks.
\end{abstract}

\section{Categories and Subject Descriptors}

D.2 [Software]: Software Engineering

; D.2.4 [Software/Program Verification]: [validation]

\section{General Terms}

Algorithms, Languages, Verification

\section{Keywords}

Nonblocking Synchronization, C++, Semantic Parallelization, Autonomous Space Software

\section{INTRODUCTION}

In this work we describe the design, implementation, and application of a first concurrency and time centered framework for verification and semantic parallelization of real-

Permission to make digital or hard copies of all or part of this work for personal or classroom use is granted without fee provided that copies are not made or distributed for profit or commercial advantage and that copies bear this notice and the full citation on the first page. To copy otherwise, to republish, to post on servers or to redistribute to lists, requires prior specific permission and/or a fee. AUTONOMICS 2008, September 23-25, Turin, Italy

Copyright (C) 2008 ICST 978-963-9799-34-9

DOI 10.4108/ICST.AUTONOMICS2008.4354 time $\mathrm{C}++$ within the JPL Mission Data System Framework (MDS). MDS provides an experimental goal- and statebased platform for testing and development of autonomous real-time flight applications[18]. The end goal of the industrial project that motivated our work is to provide certification artifacts and accelerated testing of the complex software interactions in autonomous flight systems. The process of software certification establishes the level of confidence in a software system in the context of its functional and safety requirements. A software certificate contains the evidence required for the system's independent assessment by an authority having minimal knowledge and trust in the technology and tools employed[6]. Providing such certification evidence may require the application of a number of software development, analysis, verification, and validation techniques[16]. The dominant paradigms for software development, assurance, and management at NASA rely on the principle "test-what-you-fly and fly-what-you-test". This methodology had been applied in a large number of robotic space missions at the Jet Propulsion Laboratory. For such missions, it has proven suitable in achieving adherence to some of the most stringent standards of man-rated certification such as the DO-178B[21], the Federal Aviation Administration (FAA) software standard. Its Level A certification requirements demand $100 \%$ coverage of all high and low level assurance policies. Some future space exploration projects such as the Mars Science Laboratory (MSL), Project Constellation, and the development of the the Crew Launch Vehicle (CLV) and the Crew Exploration Vehicle (CEV) suggest the engineering of some of the most complex man-rated software systems. As stated in the Columbia Accident Investigation Board Report[3], the inability to thoroughly apply the required certification protocols had been determined to be a contributing factor to the loss of STS-107, Space Shuttle Columbia.

Schumann and Visser's discussion in [22] suggests that the current certification methodologies are prohibitively expensive for systems of such complexity. A detailed analysis by Lowry[16] indicates that at the present moment the certification cost of mission-critical space software exceeds its development cost. The challenges of certifying and re-certifying avionics software has led NASA to initiate a number of advanced experimental software development and testing platforms, such as the Mission Data System (MDS)[18], as well as a number of program synthesis, modeling, analysis, and verification techniques and tools, such as The JavaPathFinder[2], the CLARAty project[25], Project Golden Gate[9], The New Millenium Architecture Prototype (New- 
MAAP)[8]. The high cost and demands of man-rated certification have motivated the experimental development of several accelerated testing platforms[1]. A great number of the experimental faster-than-real-time flight software simulators require the parallelization of previously sequential real-time algorithms. In this work we present the design and implementation of a first concurrency and time centered framework for verification and semantic parallelization of real-time $\mathrm{C}++$ within the JPL Mission Data System Framework. Our notion of semantic parallelization implies the thread-safe concurrent execution of system algorithms that utilize shared data, based on the application's semantics and invariants. As a practical industrial-scale application, we demonstrate the parallelization and verification of the MDS' Goal Networks, a critical component of the JPL's Mission Data System.

\section{CHALLENGES FOR MISSION CRITICAL AUTONOMOUS SOFTWARE}

In [17] Perrow studies the risk factors in the modern high technology systems. His work identifies two significant sources of complexity in modern systems: interactions and coupling. The systems most prone to accidents are those with complex interactions and tight coupling. With the increase of the size of a system, the number of functions it has to serve, as well as its interdependence with other systems, its interactions become more incomprehensible to human and machine analysis and this can cause unexpected and anomalous behavior. Tight coupling is defined by the presence of time-dependent processes, strict resource constraints, and little or no possible variance in the execution sequence. Perrow classifies space missions in the riskiest category since both hazard factors are present. In this work, we argue that the notions of concurrency and time are the most critical elements in the design and implementation of an embedded autonomous space system. According to a study on concurrent models of computation for embedded software by Lee and Neuendorffer[14], the major contributing factors to the development and design complexity of such systems are the underlying sequential memory models and the lack of first class representation of the notions of time and concurrency in the applied programming languages.

\subsection{Parallelism and Complexity}

The most commonly applied technique for controlling the interactions of concurrent processes is the use of mutual exclusion locks. A mutual exclusion lock guarantees threadsafety of a concurrent object by blocking all contending threads trying to access it except the one holding the lock. In scenarios of high contention on the shared data, such an approach can seriously affect the performance of the system and significantly diminish its parallelism. For the majority of applications, the problem with locks is one of difficulty of providing correctness more than one of performance. The application of mutually exclusive locks poses significant safety hazards and incurs high complexity in the testing and validation of mission-critical software. Mutual exclusion locks can be optimized in some scenarios by utilizing fine-grained locks[12] or context-switching. Often due to the resource limitations of flight-qualified hardware, optimized lock mechanisms are not a desirable alternative[16]. Even for efficient locks, the interdependence of processes im- plied by the use of locks, introduces the dangers of deadlock, livelock, and priority inversion. The incorrect application of locks is hard to determine with the traditional testing procedures and a program can be deployed and used for a long period of time before the flaws can become evident and eventually cause anomalous behavior.

\subsubsection{Nonblocking Synchronization}

To achieve higher safety and enhance the performance of our implementation, we consider the application of lock-free synchronization. As defined by Herlihy[11], a concurrent object is non-blocking (lock-free) if it guarantees that some process in the system will make progress in a finite amount of steps. Non-blocking algorithms do not apply mutually exclusive locks and instead rely on a set of atomic primitives supported by the hardware architecture. The most ubiquitous and versatile data structure in the ISO $\mathrm{C}++$ Standard Template Library [23] is vector, offering a combination of dynamic memory management and constant-time random access. In our framework for verification and semantic parallelization of real-time $\mathrm{C}++$ we utilize the design of the first lock-free design and implementation of a dynamicallyresizable array in ISO $\mathrm{C}++[5]$. It provides linearizable operations, disjoin-access parallelism for random access reads and writes, lock-free memory allocation and management, and fast execution.

\subsection{Motivation and Contributions}

As discussed by Lowry[16], in July 1997 The Mars Pathfinder mission experienced a number of anomalous system resets that caused an operational delay and loss of scientific data. The follow-up study identified the presence of a priority inversion problem caused by the low-priority meteorological process blocking the the high-priority bus management process. It has been determined that it would have been impossible to detect the problem with the black box testing applied at the time to derive the certification artifacts. A more appropriate priority inversion inheritance algorithm had been ignored due to its frequency of execution, the realtime requirements imposed, and its high cost incurred on the slower flight-qualified computer hardware. The subtle interactions in the concurrent applications of the modern aerospace autonomous software are of critical importance to the system's safety and operation.

Despite the challenges in debugging and verification of the system's concurrent components, the existing certification process[21] does not provide guidelines at the level of detail reaching the development, application, and testing of concurrent programs. This is largely due to the process-oriented nature of the current certification protocols and the complexity and high level of specialization of the aerospace autonomous embedded applications. In the near future, NASA plans to deploy a number of diverse vehicles, habitats, and supporting facilities for its imminent missions to the Moon, Mars and beyond. The large array of complex tasks that these systems would have to perform implies their high level of autonomy. In [18] Rasmussen et al. suggest that the challenges for these systems' control is one of the most demanding tasks facing NASA's Exploration Systems Mission Directorate. Some of the most significant challenges that the authors identify are managing a large number of tightlycoupled components, performing operations in uncertain remote environments, enabling the agents to respond and re- 
cover from anomalies, guaranteeing the system's correctness and reliability, and ensuring effective communication across the system's components. In the rest of the paper we describe the definition, design, and implementation of a first concurrency and time centered framework for verification and semantic parallelization of autonomous flight software within the JPL's MDS Framework. We integrate a nonblocking vector in our parallel implementation of the Mission Data System's Temporal Constraint Network Library (TCN) in order to achieve higher thread safety and boost the performance of the MDS Goal Networks component. We demonstrate how to specify, model, and formally verify the TCN algorithms and their semantic invariants. Based on our formal models and the application's semantics, we derive a technique for automatic and semantic parallelization of the TCN library's constraint propagation algorithm.

\section{TEMPORAL CONSTRAINT NETWORKS}

A Temporal Constraint Network (TCN) defines the goaloriented operation of a control system in the context of a system under control. The Temporal Constraint Networks (TCN) application is at the core of the Jet Propulsion Laboratory's Mission Data System (MDS)[18] state-based and goal-oriented unified architecture for testing and development of mission software. The framework's state- and model-based methodology and its associated systems engineering processes and development tools have been successfully applied on a number of test applications including the physical rovers Rocky 7 and Rocky 8 and a simulated Entry, Descent, and Landing (EDL) component for the Mars Science Laboratory mission. A TCN consists of a set of temporal constraints (TCs) and a set of time points (TPs). In this model of goal-driven operation, a time point is defined as an interval of time when the configuration of the system is expected to satisfy a property predicate. The width of the interval corresponds to the temporal uncertainty inherent in the satisfaction of the predicate. Similarly, temporal constraints have an associated interval of time corresponding to the acceptable bounds on the interactions between the control system and the system under control during the performance of a specific activity. A TCN graph topology represents a snapshot at a given time of the known set of activities the control system has performed so far, is currently engaged in, and will be performing in the near future up to the horizon of the elaborated plan initially created as a solution for a set of goals. The topology of a temporal constraint network must satisfy a number of invariants.

(a) A TCN is a directed acyclic graph where the edges represent the set of all time points $\left(S_{t p s}\right)$ and the vertices the set of all temporal constraints $\left(S_{t c s}\right)$

(b) For each time point $T P_{i} \in S_{t p s}$, there is a set of temporal constraints that are immediate successors $\left(S_{\text {succ }_{i}}\right)$ of $T P_{i}$ and a set, $S_{\text {pred }_{i}}$, consisting of all of $T P_{i}$ 's immediate predecessors

(c) Each temporal constraint $T C_{j} \in S_{t c s}$ has exactly one successor $T P_{s u c c_{j}}$ and one predecessor $T P_{\text {pred }_{j}}$

(d) For each pair $\left\{T P_{i}, T C_{j}\right\}$, where $T P_{i} \equiv T C_{\text {succ }_{j}}, T C_{j} \in$ $S_{\text {pred }_{i}}$ must hold. The reciprocal invariant must also be valid, namely for each pair of $\left\{T P_{i}, T C_{j}\right\}$ such that $T P_{i} \equiv T C_{\text {pred }_{j}}, T C_{j} \in S_{\text {succ }_{i}}$ (e) The firing window of a time point $T P_{i} \in S_{t p s}$ is represented by the pair of time instances $\left\{T P_{\text {min }_{i}}, T P_{\text {max }_{i}}\right\}$. Assuming that the current moment of time is represented by $T_{\text {now }}$, then $T P_{\text {min }_{i}} \leq T_{\text {now }} \leq T P_{\text {max }_{i}}$, for every $T P_{i} \in S_{t p s}$.

General-purpose programming languages lack the capabilities to formally specify and check domain-specific design constraints. Direct representation and verification of the TCN invariants in the implementation source code would result in a slow and cumbersome solution. However, any implementation (in $\mathrm{C}++$, Java or another programming language) must operate under the assumptions that the basic TCN invariants are satisfied. Thus, prior to implementing a solution to the TCN constraint propagation problem, it is necessary to guarantee the correctness and consistency of the topology of the goal network.

\section{A FRAMEWORK FOR VERIFICATION AND SEMANTIC PARALLELIZATION OF REAL-TIME C++ IN MDS}

In this section we describe the design, implementation, and practical application of our framework for verification and semantic parallelization of real-time $\mathrm{C}++$ within JPL's MDS Framework (Figure 1). The input to the framework is the MDS mission planning and execution module that is based on the definition of temporal constraint networks. At the core of the most recent implementations at JPL of this critical module is an optimized iterative algorithm for the real-time propagation of temporal constraints, developed and described by Lou in [15]. Constraint propagation poses performance challenges and speed bottlenecks due to the algorithm's frequent execution and the necessary real-time update of the goal network's topology. The end goal of our work is, given the implementation of the optimized iterative propagation scheme and the topology of a particular goal network, to establish the correctness of the core TCN semantic invariants (see Section 3) and automatically derive an implementation that can be executed concurrently on one of the JPL's experimental testbeds for accelerated testing[1]. Our approach for achieving concurrent execution is based on the idea of identifying Time Phases within a goal network, which allow the semantic parallelization of the constraint propagation algorithm. In this work, we define semantic parallelization as the thread-safe concurrent execution of an algorithm (whose operation is dependent on shared data), derived from the application's semantics and invariants. In the following sections we describe how we reach our goal of verification and semantic parallelization of the mission planning and control module by constructing and executing a formal verification model in Alloy[13] that represents the implementation's core semantics and functionality. We refine a formal modeling and analysis methodology, initially suggested by Rouquette[20], that helps us analyze the logical properties of the goal network model and automatically derive a meta-model for our parallel solution.

\subsection{The Problem of TCN Constraint Propa- gation}

A classic solution to the problem of constraint propagation in TCN is the direct application of Floyd-Warshall's all-pairs-shortest-path algorithm[4], offering a complexity of 


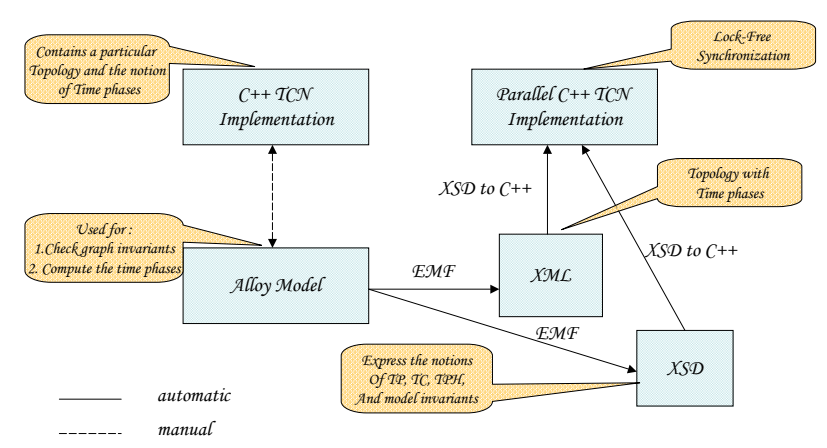

Figure 1: A Framework for Verification and Semantic Parallelization

$O\left(N^{3}\right)$, where $N$ is the number of time points in the TCN topology. Since, by definition, the goal of the TCN propagation algorithm is to compute the real-time values of the network's temporal constraints, the algorithm is frequently executed and, given the massive scale of a real world goal network, can cause significant bottleneck for the overall system's performance. In [15], Lou describes an innovative and effective TCN propagation scheme with a complexity close to linear. Lou's TCN propagation is based on the concept of alternating forward and backward propagation passes. A forward pass updates the time interval at each time point by considering only its incoming temporal constraints (Algorithm 1). Similarly, a backward pass recomputes the time windows at each time point by considering only its outgoing temporal constraints (Algorithm 2). The scheme utilizes a shared container, named a propagation queue, to keep track of all time points whose successor time points' windows are about to be updated next (during a forward pass) and all time points whose predecessor time points' windows are about to be updated next (during a backward pass). A forward pass begins by selecting all time points with no predecessors and inserts them into the propagation queue. A backward pass begins by selecting all time points with no successors and inserts them into the propagation queue. Each iteration is carried out until:

(a) An iteration completes without updating any temporal constraints (thus indicating that there are no more updates to be performed during the pass). In this case, the TCN topology is considered to be temporally consistent.

(b) The iteration has stumbled upon a time window of negative value and the algorithm terminates with the outcome of having a temporally inconsistent network.

As stated by Lou[15], prior to the execution of the optimized propagation scheme, it is critical to guarantee the validity of the core TCN invariants for the topology of the particular goal network. For example, the propagation scheme operates under the assumption that the goal network graph is cycle free. Should there be cycles, the propagation would enter into an endless loop.

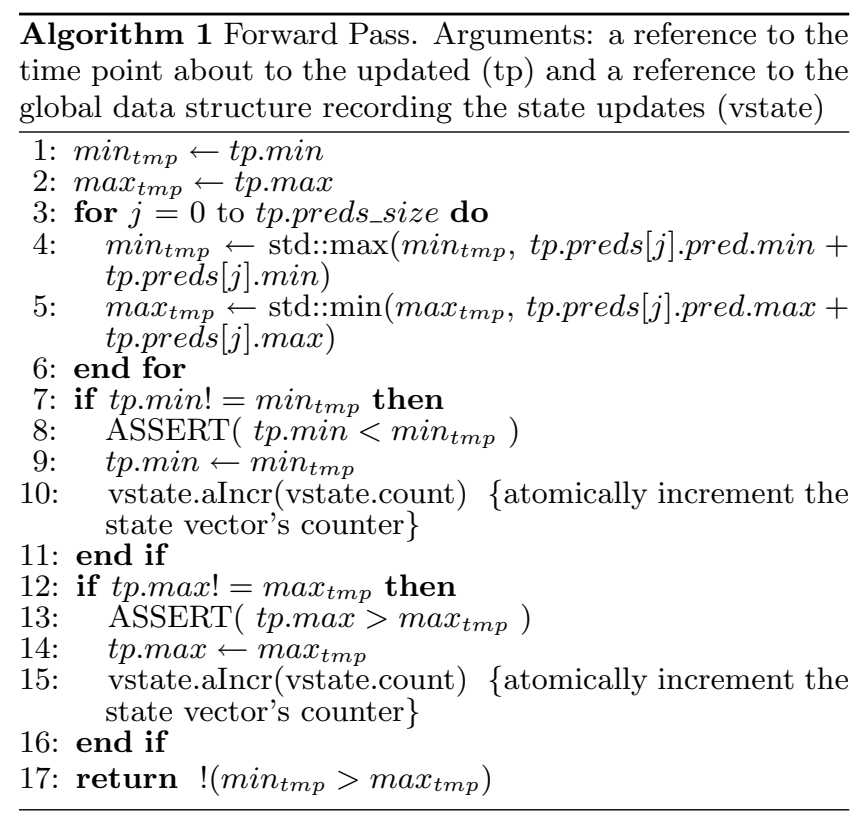

Algorithm 2 Backward Pass. Arguments: a reference to the time point about to the updated (tp) and a reference to the global data structure recording the state updates (vstate)

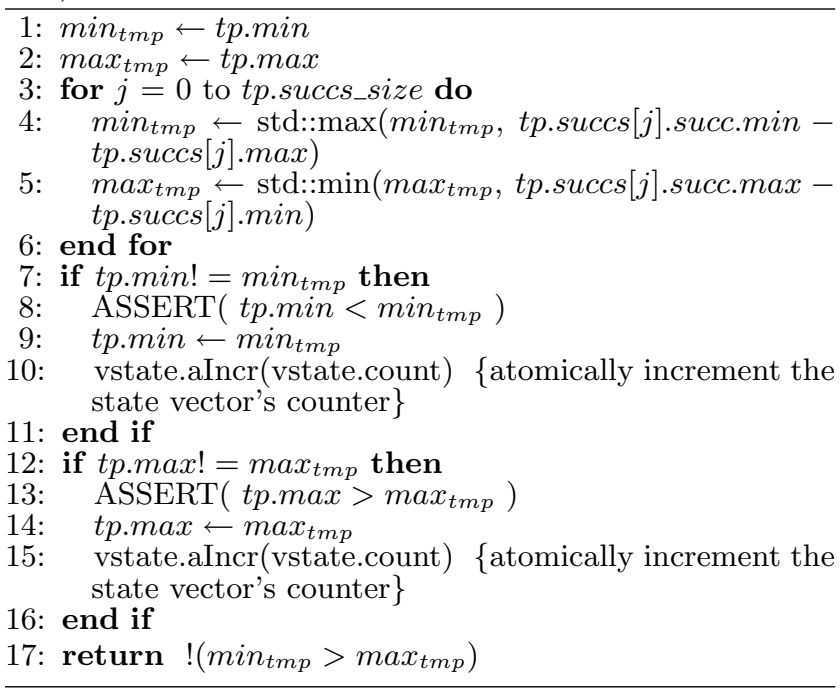

\subsection{Modeling, Formal Verification, and Auto- matic Parallelization}

Alloy[13] is a lightweight formal specification and verification tool for the automated analysis of user-specified invariants on complete or partial models. The Alloy Analyzer is implemented as a front-end, performing the role of a model-finder, to a boolean SAT-solver. Formal verification and modeling of JPL's flight software has been previously demonstrated to be effective and successful by Holzmann[10]. We use the Alloy specification language[13] to formally represent and check the semantics of the temporal constraint networks library (Algorithm 3) and its main 
tionally expensive when applied to the parallel propagation algorithm. Given the frequent real-time changes in the graph topology, employing a thread per iteration for the computations of each time phase comes at a prohibitive cost. To avoid this problem, we have incorporated in our design the application of the Intel tasks from the Threading Building Blocks Library[12]. Our experiments indicate that the Intel tasks provide lowcost overhead when applied in the concurrent execution of the forward and backward passes of the propagation scheme.

(2) Allowing fast and safe access to the shared data. The parallel algorithm requires the safe and efficient concurrent synchronization of its shared data: the propagation queue and the vector containing control data (reflecting the updates during an iteration). By the definition of our algorithm, the propagation queue is synchronized by allowing only disjoint-access writes. While the access to the shared vector is less frequent, its concurrent synchronization is more challenging since we do not have a guarantee that the concurrent writes would be disjoint. The application of mutual exclusion locks is a possible but likely an ineffective solution due to the risks of deadlock, livelock, and priority inversion. Moreover, the interdependency of processes implied by the use of locks diminishes the parallelism of a concurrent system. A lock-free object guarantees that within a set of contending processes, there is at least one process that will make progress within a finite number of steps. We have employed the implementation of the lock-free vector described in [5] in order to meet our goals for thread-safe and effective non-blocking synchronization. The lockfree vector provides the functionality of the popular STL $\mathrm{C}++$ vector as well as linearizable and safe operations with complexity of $O(1)$ and fast execution (outperforming the STL vector protected by a mutex by a factor of 10 or more).

A number of graph properties, in a particular TCN topology, have a significant impact on the application and performance of the parallel propagation scheme. We expect better performance (with respect to the sequential propagation scheme) when:

(1) The computational load per time point is high. This is the case of a real-world massive-scale goal network. For instance, instructing the Mars Science Laboratory to autonomously find its way in a Martian crater, probe the soil, capture images, and communicate to Mission Control will result in a goal network containing tens or hundreds of thousands of time points. In a small experimental graph topology with a low computational cost per time point (such as a few arithmetic operations), a single processor computation will perform best (when we take into account the parallelization overhead).

(2) Time phases with large number of time points: a topology implying a sequential ordering of the planned events will not benefit from a parallel propagation scheme. The parallel propagation algorithm is beneficial to goal networks representing a large number of highly interactive concurrent system processes.

\subsection{Framework Application for Accelerated Test- ing}

The presented design and implementation of our parallel propagation technique enable the incorporation of the optimized propagation approach described by Lou[15] in an experimental framework for accelerated testing currently still under development at NASA. Accelerated testing platforms suggest a paradigm shift in the certification process employed by NASA from system testing with the actual flight hardware and software to accelerated cost-effective certification using hardware simulators and distributed software implementations (Figure 3). Such frameworks aim fasterthan-real-time testing and analysis of the complex software interactions in JPL's autonomous flight systems. A number of these platforms require automated refactoring of previously sequential code into modular parallel implementations. Preliminary results reported in academic work[1] as well as experience reports from a number of commercial tools (such as Simics by Virtutech and ADvantage BEACON by Applied Dynamics International) suggest the possible speedup of the flight system testing by a significant factor. We have followed Rouquette's methodology[20] that suggests the application of formal modeling and validation techniques that provide certification evidence for a number of functional dependencies in order to compensate for the added hazards in establishing the fidelity of the simulators. Due to the incom-

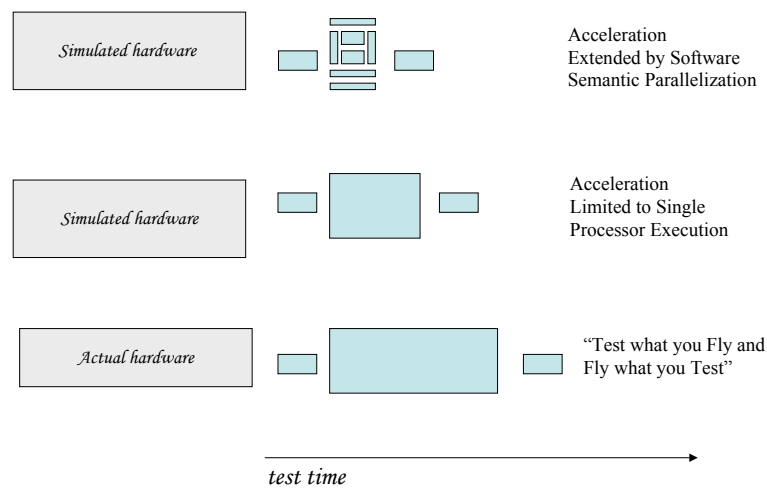

Figure 3: Testing Scenarios of Mission Software

plete status of the accelerated testing framework as well as the lack of the actual flight hardware, it is difficult to measure a priori the effect of our parallel propagation scheme in achieving acceleration (with respect to the execution on the actual flight hardware) in the process of flight software testing. To gain insight of the possible performance gains and the algorithm's behavior we ran performance tests on a conventional Intel IA-32 SMP machine with two $2.0 \mathrm{GHz}$ processor cores with 1GB shared memory and 4 MB L2 shared cache running the MAC OS 10.5.1 operating system. In our performance analysis we have measured the execution time in seconds of two versions of our parallel propagation algorithm (one applying mutually exclusive locks and the other relying on nonblocking synchronization) and the orig- 
inal sequential scheme presented by Lou[15]. In the experiments (Figure 4), we have generated a number of TCN graph topologies (each consisting of 4 to 8 Time Phases), in a manner similar to the pseudo-random graph generation methodology described in [7]. In the presented results on Figure 4 the $x$-axis represents the average measured execution time (in seconds) of each propagation scheme and the $y$-axis represents the number of time points in the exponentially increasing graph size (starting with a graph of 20000 TPs and reaching a TCN having 160000 TPs). In the

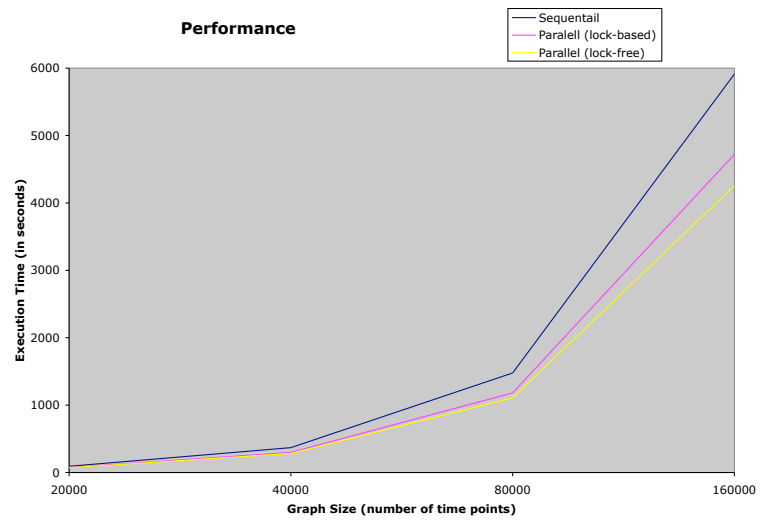

Figure 4: Performance Analysis. x-axis represents the number of TPs in each experimental TCN topology, yaxis represents the execution time in seconds of each of the three propagation algorithms

experimental setup we observed that the parallel propagation algorithm offers effective execution and a considerable speedup in all scenarios on our dual-core platform. We measured performance acceleration reaching $28 \%$ in the case of the nonblocking implementation and $20 \%$ for our algorithm relying on mutually exclusive locks. Lock-free algorithms deliver significant speedup in applications utilizing shared data under high contention[5]. In a scenario like our parallel TCN propagation scheme with medium or low contention on the shared data, besides safety and prevention of priority inversion and deadlock, a lock-free implementation can guarantee better scalability. As our experimental results suggest, the gains from the lock-fee implementation gradually progress and we observe better scalability with respect to the blocking propagation scheme. Based on the experimental results, we expect that the integration of our parallel propagation algorithm in the accelerated testing framework (consisting of several dozen processing units) will deliver significant benefits in reaching cost-effective and reliable flight software certification of control modules based on massive real-world goal networks.

\section{CONCLUSION}

The notions of time and concurrency are of critical importance for the design and development of autonomous space systems. The current certification methodologies do not reach the level of detail of providing guidelines for the development and validation of concurrent and real-time software. The increasing number of complex interactions and tight coupling of the future autonomous space systems pose significant challenges for their development and man-rated certification. A number of platforms for accelerated testing suggest a paradigm shift by applying a combination of modeling and verification methods, code generation tools, and software parallelization for establishing a cost-effective and reliable certification process. In the light of the challenges posed by the design and development of these highly experimental approaches, we presented in this work a first time- and concurrency-centered framework for validation and semantic parallelization of real-time $\mathrm{C}++$ within JPL's MDS Framework. We demonstrated the application of our framework in the validation of the semantic invariants of the Temporal Constraint Network Library. Temporal constraint networks are at the core of the mission planning and control architecture of the Mission Data System framework. In addition, we presented an approach for automatic semantic parallelization of the propagation scheme establishing the consistency of the temporal constraints in a goal network. Our parallel propagation scheme is based on the identification of time phases within a goal network and is implemented through the application of model transformation and formal analysis techniques to the model specifications of the TCN semantics. We have relied on innovative lock-free synchronization techniques to achieve better performance and higher safety of our parallel implementation. Our preliminary tests indicate that our parallel propagation approach, upon integration in the accelerated testing framework, can support cost-effective and reliable flight software certification of control modules based on massive real-world goal networks. In our future work we plan to focus on developing a component for automatic derivation of the model specification directly from implementation source code. This can be accomplished by utilizing the high-level internal program representation and the analysis tools provided by The Pivot[24], a framework for static analysis and transformations in $\mathrm{C}++$.

\section{ACKNOWLEDGEMENTS}

We thank Kirk Reinholtz and David Wagner from the Jet Propulsion Laboratory for their collaboration and the provided directions on this work.

\section{REFERENCES}

[1] B. Boehm, J. Bhuta, D. Garlan, E. Gradman, L. Huang, A. Lam, R. Madachy, N. Medvidovic, K. Meyer, S. Meyers, G. Perez, K. Reinholtz, R. Roshandel, and N. Rouquette. Using Empirical Testbeds to Accelerate Technology Maturity and Transition: The SCRover Experience. In ISESE '04: Proceedings of the 2004 International Symposium on Empirical Software Engineering, pages 117-126, Washington, DC, USA, 2004. IEEE Computer Society.

[2] G. Brat, D. Drusinsky, D. Giannakopoulou, A. Goldberg, K. Havelund, M. Lowry, C. Pasareanu, A. Venet, R. Washington, and W. Visser. Experimental Evaluation of Verification and Validation Tools on Martian Rover Software. In Formal Methods in Systems Design Journal, September 2005.

[3] Columbia Accident Investigation Board. Columbia Accident Investigation Board Report Volume 1. 
[4] T. H. Cormen, C. E. Leiserson, R. L. Rivest, and C. Stein. Introduction to algorithms. MIT Press, Cambridge, MA, USA, 2001.

[5] D. Dechev, P. Pirkelbauer, and B. Stroustrup. Lock-Free Dynamically Resizable Arrays. In A. A. Shvartsman, editor, OPODIS, volume 4305 of Lecture Notes in Computer Science, pages 142-156. Springer, 2006.

[6] E. Denney and B. Fischer. Software Certification and Software Certification Management Systems. In SoftCement05. Proceedings of the 2005 ASE Workshop on Software Certificate Management, 2005.

[7] R. P. Dick, D. L. Rhodes, and W. Wolf. Tgff: task graphs for free. In CODES/CASHE '98: Proceedings of the 6th international workshop on Hardware/software codesign, pages 97-101, Washington, DC, USA, 1998. IEEE Computer Society.

[8] D. Dvorak. Challenging encapsulation in the design of high-risk control systems. In Proceedings of the 17th ACM Conference on Object-Oriented Programming, Systems, Languages, and Applications (OOPSLA'02), 2002.

[9] D. Dvorak, G. Bollella, T. Canham, V. Carson, V. Champlin, B. Giovannoni, M. Indictor, K. Meyer, A. Murray, and K. Reiinholtz. Project Golden Gate: Towards Real-Time Java in Space Missions. In In the Proceedings of the 7th IEEE International Symposium on Object-Oriented Real-Time Distributed Computing (ISORC'04), 2004.

[10] R. Gluck and G. Holzmann. Using the spin model checker for flight software verification. In Proceedings of the 2002 IEEE Aerospace Conference, 2002.

[11] M. Herlihy. A methodology for implementing highly concurrent data structures. In PPOPP '90: Proceedings of the second ACM SIGPLAN symposium on Principles \& practice of parallel programming, pages 197-206, New York, NY, USA, 1990. ACM Press.

[12] Intel. Reference for Intel Threading Building Blocks, version 1.0, April 2006.

[13] D. Jackson. Software Abstractions: Logic, Language and Analysis. The MIT Press, 2006.

[14] E. Lee and S. Neuendorffer. Concurrent Models of computation for Embedded Software. In IEEE Proceedings on Computers and Digital Techniques, March 2005.

[15] J. Lou. An Efficient Algorithm for Propagation of Temporal Constraint Networks. NASA Tech Brief Vol. 26 No. 4 from JPL New Technology Report NPO-21098, April 2002.

[16] M. R. Lowry. Software Construction and Analysis Tools for Future Space Missions. In J.-P. Katoen and P. Stevens, editors, TACAS, volume 2280 of Lecture Notes in Computer Science, pages 1-19. Springer, 2002.

[17] C. Perrow. Normal Accidents. Princeton University Press, September 1999.

[18] R. Rasmussen, M. Ingham, and D. Dvorak. Achieving Control and Interoperability Through Unified Model-Based Engineering and Software Engineering. In AIAA Infotech at Aerospace Conference, 2005.

[19] G. D. Reis and B. Stroustrup. Specifying C++ Concepts, ISO WG21 N1886, 2005.

[20] N. Rouquette. Analyzing and verifying UML models with OCL and Alloy. EclipseCon 2008, 2008.

[21] RTCA. Software Considerations in Airborne Systems and Equipment Certification (DO-178B), 1992.

[22] J. Schumann and W. Visser. Autonomy Software: V\&V Challenges and Characteristics. In Proceedings of the 2006 IEEE Aerospace Conference, 2006.

[23] B. Stroustrup. The C++ Programming Language.
Addison-Wesley Longman Publishing Co., Inc., Boston, MA, USA, 2000.

[24] B. Stroustrup and G. D. Reis. Supporting SELL for High-Performance Computing. In Proceedings of the International Workshop on Languages and Compilers for Parallel Computing, LCPC 2005, 2005.

[25] R. Volpe, I. Nesnas, T. Estlin, D. Mutz, R. Petras, and H. Das. The CLARAty Architecture for Robotic Autonomy. In IEEE Aerospace Conference, March 2001 . 